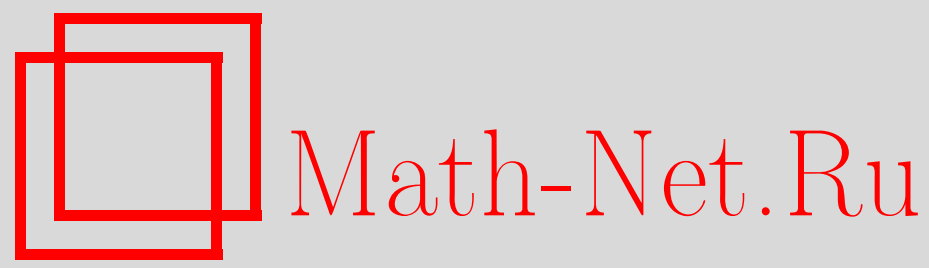

Ю. В. Болотников, Частично разложимые и вполне неразложимые неотрицательные матрицы, Матем. заметки, 1996, том 59, выпуск 5, 643-662

DOI: https://doi.org/10.4213/mzm1759

Использование Общероссийского математического портала MathNet.Ru подразумевает, что вы прочитали и согласны с пользовательским соглашением

http://www. mathnet.ru/rus/agreement

Параметры загрузки:

IP : 44.207 .124 .84

26 апреля 2023 г., 10:12:07

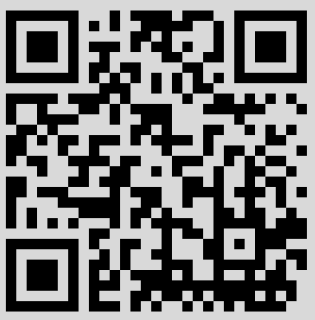




\title{
ЧАСТИЧНО РАЗЛОЖИМЫЕ И ВПОЛНЕ НЕРАЗЛОЖИМЫЕ НЕОТРИЦАТЕЛЬНЫЕ МАТРИЦЫ
}

\author{
Ю.В. Болотников, В.Е. Тараканов
}

Мы рассматриваем неотрицательные матрицы размера $m \times n, m \leqslant n$, элементы которых принадлежат фиксированному множеству $R_{q}^{+}$из $q$ неотрицательных чисел, включающему нуль. Если в такой матрице $A$ имеются нулевые подматрицы размера $r \times s$ и $r+s=t$, то максимальное из таких чисел $t$ назьвается нульностью матрицы $A$ (см. [1]).

В $\S 1$ мы находим асимптотическое при $n \rightarrow \infty$ разложение для числа матриц нульности $t$ при $t>n$, а также для числа полу-вполне неразложимых матриц $(m<n, t<n)$, играюших существенную роль при изучении матриц с нулевым перманентом (см. [2]). Для числа $f(m, n)$ матриц с нулевым перманентом получено асимптотическое разложение без использования принципа включения-исключения и неравенств Бонферрони (как было в [2]).

В 22 получена асимптотика для числа вполне неразложимых матрищ порядка $n$.

Отметим, что наши рассмотрения включают (при $q=2$ ) случай $(0,1)$-матриц, и нами тем самым установлена асимптотика числа частично разложимых и вполне неразложимых $(0,1)$-матриц размера $m \times n$, $m \leqslant n$, при $n \rightarrow \infty$.

\section{§1. Частично разложимые матрицы}

Пусть $R_{q}^{+}$- фиксированное множество из $q$ неотрицательных чисел, включающее нуль. Мы рассматриваем множество $M(m, n)$ всевозможных матрищ размера $m \times n, m \leqslant n$, с элементами из $R_{q}^{+}$. Неотрицательная матрица $A \in M(m, n)$ назьвается частично разложимой, если ее нульность больше или равна $n$. Матрища, не являющаяся частично разложимой, называется полу-вполне неразложимой (см. [1]), а в

Работа второго автора вьполнена при финансовой поддержке Российского фонда фундаментальных исследований, грант № 93-011-1443.

(C) Ю. В. БолОтников, В. Е. ТАРАКАнов 
случае $m=n-$ вполне неразложимой. Мы применяем эти определения и к матрище $A$ размера $m \times n$ с $m>n$, если им удовлетворяет транспонированная матрица $A^{T}$.

Пусть $f(m, n), g(m, n), h(m, n)$ обозначают соответственно числа матриц из $M(m, n)$ с нулевьп перманентом, с перманентом, отличным от нуля, и полу-вполне неразложимых матрищ. Полагаем также $g(0, n)=$ $h(0, n) \equiv 1$ для любого натурального $n$. Обозначим $M_{t}(m, n)$ множество всех матриц нульности $t$, а $c_{t}(m, n)$ - их число. Для матрищ из $M_{t}(m, n)$ введем еще понятие типа: матрица $A \in M_{t}(m, n)$ имеет mun $s$, если она содержит нулевую подматрицу $O_{r s}$ размера $r \times s, r+s=t$, и для любой ее нулевой подматрищы $O_{u v}$ с $u+v=t$ выполнено $v \geqslant s$. Множество матриц нульности $t$ и типа $s$ обозначим $M_{t}^{(s)}(m, n)$, а их число $c_{t}^{(s)}(m, n)$. Особо выделим числа $c_{n}(m, n)$ и $c_{n}^{(s)}(m, n)$ и обозначим их $p(m, n)$ и $p^{(s)}(m, n)$. В матрице $A$ из $M_{t}^{(s)}(m, n)$ любая нулевая подматрища размера $r \times s, r+s=t$, назьвается максимальной.

В работе [2] доказано, что при $t>n$ и при $t=n, m<n$ максимальная подматрища единственна, а также получено равенство

$$
c_{t}^{(s)}(m, n)=\left(\begin{array}{c}
m \\
r
\end{array}\right)\left(\begin{array}{l}
n \\
s
\end{array}\right) q^{(m-r)(n-s)} g(n-s, r) h(m-r, s),
$$

справедливое при $t>n$ и $t=n, m<n$. Мы будем пользоваться соотношением (1) и вытекающими из него оценками, в частности, следующей:

$$
c_{t}^{(s)}(m, n) \leqslant\left(\begin{array}{l}
t \\
s
\end{array}\right)^{2} q^{m n-s(t-s)}
$$

Частично разложимая матрища размера $m \times n-$ это матрица нульности не меньше $n$, поэтому число таких матриц равно

$$
\sum_{t=n}^{m+n} c_{t}(m, n)=p(m, n)+f(m, n)
$$

В свою очередь, тип $s$ матрицы $A \in M_{t}(m, n)$ изменяется от $\max \{t-m, 1\}$ до $\min \{t-1, n\}$, поэтому

$$
c_{t}(m, n)=\sum_{s=\max \{t-m, 1\}}^{\min \{t-1, n\}} c_{t}^{(s)}(m, n) .
$$

При оценках нам понадобятся асимптотические соотношения для биномиальных коэффициентов, вытекающие при $n \rightarrow \infty$ из формулы Стирлинга:

$$
\begin{aligned}
& \left(\begin{array}{c}
n \\
n / 2
\end{array}\right) \sim \sqrt{\frac{2}{\pi} \frac{2^{n}}{\sqrt{n}}} \\
& \left(\begin{array}{c}
n \\
\sqrt{n}
\end{array}\right) \sim \frac{1}{\sqrt{2 \pi e \sqrt{n}}}(e \sqrt{n})^{\sqrt{n}} .
\end{aligned}
$$


Мы будем оценивать число матриц нульности $t$ при $t \geqslant n$. Оценим сначала при достаточно большом $n$ и некоторой константе $u$ общее число матриц нульности не меньше $t+u$. Предварительно докажем одно вспомогательное утверждение.

Лемма 1. Пусть $m=m(n) u t=t(n)$, где $m \leqslant n \leqslant t \leqslant m+n$, $n=1,2, \ldots,-$ две последовательности натуральных чисел, a $u, v-$ два натуральных числа таких, что $v+4 \leqslant u \leqslant m+n-t$. Пусть $t_{1}=t+u$, a $p=n-v$. Тогда при $n \rightarrow \infty$

$$
\left(\sum_{s=t_{1}-m}^{n}\left(\begin{array}{c}
t_{1} \\
s
\end{array}\right)^{2} q^{m n-s\left(t_{1}-s\right)}\right) / q^{m n-n(t-p-1)}=O\left(q^{-n}\right) .
$$

ДокаЗАТЕЛЬСтво. Обозначим $S$ сумму в левой части (7). Ясно, что $m n-s\left(t_{1}-s\right)$ достигает максимума при $s=n$. Поэтому, ввиду (5), для достаточно больших $n$

$$
S \leqslant\left(m+n-t_{1}+1\right) \frac{2}{\pi} \frac{2^{2 t_{1}}}{t_{1}} q^{m n-n\left(t_{1}-n\right)} .
$$

Следовательно,

$$
\frac{S}{q^{m n-n(t-p-1)}} \leqslant q^{n(t-p-1)-n\left(t_{1}-n\right)+2 t_{1}},
$$

так как $m+n-t_{1}+1 \leqslant t_{1}$. Теперь замечаем, что при наших предположениях показатель степени в правой части неравенства не превьшает $-n$. Тем самьм получаем утверждение леммы.

Лемма 2. Пусть $u, v-$ натуральные числа, $u \geqslant v+4$. При $t \geqslant n$, $u \leqslant m+n-t$ справедлива при $n \rightarrow \infty$ асимптотическая оченка

$$
\left(\sum_{t_{1}=t+u}^{m+n} c_{t_{1}}(m, n)\right) / q^{m n-n(t-n+v-1)}=O\left(\frac{m}{q^{n}}\right) .
$$

ДоКАЗАТЕЛЬСтво. Рассмотрим для каждого $c_{t_{1}}(m, n)$, где $t+u \leqslant$ $t_{1} \leqslant m+n$, представление (4). Утверждение получается, если воспользоваться неравенством (2) и применить затем оценку (7) к каждому из слагаемых в получившейся сумме. Таким образом,

$$
\frac{c_{t_{1}}(m, n)}{q^{m n-n(t-n+v-1)}}=O\left(q^{-n}\right)
$$

для каждого из чисел $t+u, t+u+1, \ldots, m+n$, и мы приходим к (8). Лемма доказана. 
Перейдем к оценкам чисел $c_{t}(m, n)$ при значениях $t$, достаточно близких к $n$. Пусть $m=m(n)$ и $t=t(n), n=1,2, \ldots,-$ две последовательности натуральных чисел. Будем говорить, что $m, n$ и $t$ yдовлетворяют условию $(*)$, если вьполнены следующие условия:

$$
m(n) \leqslant n \leqslant t(n)
$$

и для достаточно больших $n$

$$
n>t-\varepsilon(t) \sqrt{t}
$$

где $\varepsilon(t) \rightarrow \varepsilon, 0<\varepsilon<1$, при $n \rightarrow \infty$. Из (9) и (10), очевидно, следует, что $n \sim t$. Заметим, что если $m=m(n) \leqslant n, t=t(n)=n+u$, где $u$ - целое неотрицательное число, то $m, n, t$ удовлетворяют условию $(*)$.

$\mathrm{B}$ дальнейшем мы используем иногда обозначения вида $\sum_{s=a}^{b}$, где $a$ и $b$ могут быть не целыми числами, понимая под этим суммирование по всем целым числам в пределах от $a_{0}$ до $b_{0}$, где $a_{0}, b_{0}$ - ближайшие к $a$ и $b$ целые числа.

При оценках $c_{t}(m, n)$ мы будем сумму в правой части формулы (4) при $\sqrt{t}<m \leqslant t-\sqrt{t}$ разбивать на две части - от $t-m$ до $t-\sqrt{t}-1$ и от $t-\sqrt{t}$ до $\min \{n, t-1\}$; а при $m>t-\sqrt{t}$ на три части - от $\max \{t-m, 1\}$ до $\sqrt{t}-1$, от $\sqrt{t}$ до $t-\sqrt{t}-1$ и от $t-\sqrt{t}$ до $\min \{n, t-1\}$. Для оценки этих сумм докажем еще несколько вспомогательных утверждений.

Лемма 3. Пусть $m, n, t$ удовлетворяют условию (*), v - натуральное число и $n-v=p_{1}$. Тогда при $n \rightarrow \infty$

$$
\sum_{s=\beta}^{p_{1}}\left(\begin{array}{l}
t \\
s
\end{array}\right)^{2} q^{m n-s(t-s)}=o\left(q^{m n-n\left(t-p_{1}-1\right)}\right),
$$

әде $p_{1} \geqslant \beta \geqslant t-\sqrt{t}$.

Далее, пусть $m>t-\sqrt{t}$ и $p-$ челое число со свойствами $t-\sqrt{t}<$ $p \leqslant m, p \leqslant p_{1}$. Тогда при $n \rightarrow \infty$ справедливо также

$$
\sum_{s=t-p}^{\sqrt{t}}\left(\begin{array}{l}
t \\
s
\end{array}\right)^{2} q^{m n-s(t-s)}=o\left(q^{m n-n\left(t-p_{1}-1\right)}\right) .
$$

ДокАЗАТЕЛЬСТво. Положим $\varphi(s)=s(t-s), s=1,2, \ldots, t$. Обозначим $S_{1}$ левую часть (11). Тогда

$$
S_{1} \leqslant(\sqrt{t}+n-t)\left(\begin{array}{l}
t \\
s
\end{array}\right)^{2} q^{m n-\varphi\left(p_{1}\right)} .
$$


Ввиду (6) для достаточно больших $n$ получаем

$$
S_{1} \leqslant(\sqrt{t}+n-t) \frac{1}{2 \pi e \sqrt{t}}(e \sqrt{t})^{2 \sqrt{t}} q^{m n-\varphi\left(p_{1}\right)}
$$

Так как $\sqrt{t}+n-t \leqslant \sqrt{t}$, то

$$
\frac{S_{1}}{q^{m n-n\left(t-p_{1}-1\right)}}<(e \sqrt{t})^{2 \sqrt{t}} q^{n\left(t-p_{1}-1\right)-\varphi\left(p_{1}\right)}
$$

Далее, по условиям леммы

$$
n\left(t-p_{1}-1\right)-p_{1}\left(t-p_{1}\right)<-n+v \sqrt{t}+v^{2}
$$

Учитьвая, что в наших условиях $t \sim n$, видим, что логарифм правой части в (13) при $n \rightarrow \infty$ стремится к $-\infty$. Поэтому из (13) следует (11).

Пусть теперь $p$ - целое число со свойствами $t-\sqrt{t}<p \leqslant m, p \leqslant p_{1}$. Обозначим $S_{2}$ левую часть в (12). Аналогично предыдущему получаем

$$
\frac{S_{2}}{q^{m n-n\left(t-p_{1}-1\right)}} \leqslant(e \sqrt{t})^{2 \sqrt{t}} q^{n\left(t-p_{1}-1\right)-\varphi(p)} .
$$

Нетрудно заметить, что $\varphi\left(p_{1}\right) \leqslant \varphi(p)$, так как $t / 2<t-\sqrt{t}<p \leqslant p_{1}$. Поэтому правая часть в (14) не превосходит правой части в (13), которая, как мы показали вьше, стремится к нулю при $n \rightarrow \infty$. Таким образом, из (14) следует (12). Лемма доказана.

СЛЕДСТВИЕ 1. Если $m, n, t$ удовлетворяют условию (*), то при $n \rightarrow \infty$

$$
\sum_{s=\max \{t-m, t-\sqrt{t}\}}^{n-v}\left(\begin{array}{l}
t \\
s
\end{array}\right)^{2} q^{m n-s(t-s)}=o\left(q^{m n-n(t-n)-(v-1) n}\right),
$$

әде $v$ - натуральное число такое, что $t \leqslant m+n-v$.

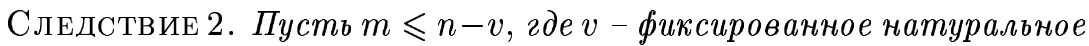
число, и $m, n, t$ удовлетворяют условию (*). Тогда при $m>t-\sqrt{t}$ имеем

$$
\sum_{s=t-m}^{\sqrt{t}}\left(\begin{array}{l}
t \\
s
\end{array}\right)^{2} q^{m n-s(t-s)}=o\left(q^{m n-n(t-n)-(v-1) n}\right),
$$

если $n \rightarrow \infty$.

Действительно, при $m>t-\sqrt{t}$ оценка (16) вытекает из утверждения леммы, если положить $p=m, p_{1}=n-v$. 
Лемма 4. Пусть $m, n, t$ удовлетворяют условию (*), $\alpha$ - челое число в интервале $[\sqrt{t}, t-\sqrt{t}-1], l(n, t)-$ произвольная линейная функиия от $n, t$ с постоянными коэффичиентами. Тогда при $n \rightarrow \infty$

$$
\sum_{s=\alpha}^{t-\sqrt{t}-1}\left(\begin{array}{l}
t \\
s
\end{array}\right)^{2} q^{m n-s(t-s)}=o\left(q^{m n-n(t-n)+l(n, t)}\right) .
$$

ДокАЗАТЕЛЬСтво. Обозначим через $S$ левую часть в (17). Тогда

$$
S \leqslant(t-\sqrt{t}))\left(\begin{array}{c}
t \\
t / 2
\end{array}\right)^{2} q^{m n-\sqrt{t}(t-\sqrt{t})},
$$

следовательно, ввиду (5)

$$
\frac{S}{q^{m n-n(t-n)+l(n, t)}} \leqslant q^{\Phi(t)},
$$

где $\Phi(t)=n(t-n)-t \sqrt{t}+t+l_{1}(n, t)$, a $l_{1}(n, t)=-l(n, t)+2 t$. Запишем

$$
\Phi(t)=-t \sqrt{t}\left(1-\frac{n}{t}, \frac{t-n}{\sqrt{t}}-\frac{1}{\sqrt{t}}-\frac{l_{1}(n, t)}{t \sqrt{t}}\right) .
$$

Очевидно, в наших условиях для всех достаточно больших $n$ выражение в скобках не меньше величины, сколь угодно близкой к положительному числу $1-\varepsilon$. Отсюда следует утверждение леммы.

Мы можем теперь вывести асимптотические оценки сверху для чисел $c_{t}(m, n), t \geqslant n$. Для удобства формулировок расширим область определения чисел $c_{t}^{(s)}(m, n)$ : положим $c_{t}^{(s)}(m, n)=0$ при $s<\max \{t-m, 1\}$.

ЛЕмма 5. Пусть $m, n, t$ удовлетворяют условию $(*) u t>n$. Тогда nрu $n \rightarrow \infty$

$$
\begin{aligned}
c_{t}(m, n) \leqslant \alpha & (m, n, t) q^{m n-n(t-n)} \\
& +\beta(m, n, t) q^{m n-n(t-n)-n}(1+o(1)),
\end{aligned}
$$

¿əe

$$
\begin{aligned}
\alpha(m, n, t)= \begin{cases}\left(\begin{array}{c}
m \\
t-n
\end{array}\right), & m<n, \\
2\left(\begin{array}{c}
n \\
t-n
\end{array}\right), & m=n,\end{cases} \\
\beta(m, n, t)=\left\{\begin{array}{l}
\left(\begin{array}{c}
m \\
t-n+1
\end{array}\right) n\left(q^{t-n+1}-1\right), \quad m \leqslant n-2, \\
\left(\begin{array}{c}
n-1 \\
t-n+1
\end{array}\right) n\left(q^{t-n+1}-1\right)+\left(\begin{array}{c}
n \\
t-n+1
\end{array}\right) q^{t-n+1}, \\
m=n-1, \\
\left(\begin{array}{c}
n \\
t-n+1
\end{array}\right) n\left[2 q^{t-n+1}-(q-1)(t-n+1)-2\right], \\
m=n .
\end{array}\right.
\end{aligned}
$$


ДоКАЗАТЕЛЬСТВо. Воспользуемся формулой (4). Введем обозначения:

$$
\begin{gathered}
\Sigma_{1}=\sum_{s=t-\sqrt{t}}^{n-2} c_{t}^{(s)}(m, n), \quad \Sigma_{2}=\sum_{s=\sqrt{t}}^{t-\sqrt{t}-1} c_{t}^{(s)}(m, n), \\
\Sigma_{3}=\sum_{s=t-n+2}^{\sqrt{t}-1} c_{t}^{(s)}(m, n) .
\end{gathered}
$$

Так как $t>n$, мы можем записать

$$
\begin{aligned}
c_{t}(m, n)= & c_{t}^{(n)}(m, n)+c_{t}^{(n-1)}(m, n)+\Sigma_{1}+\Sigma_{2} \\
& +\Sigma_{3}+c_{t}^{(t-n+1)}(m, n)+c_{t}^{(t-n)}(m, n) .
\end{aligned}
$$

Оценим слагаемые в (21). При этом для оценки $\Sigma_{1}, \Sigma_{2}, \Sigma_{3}$ применяем леммы 3 и 4 . Остальные члены в (21) оцениваем точнее, используя (1), а также следуюшие очевидные соотношения:

$$
\begin{aligned}
& g(1,1)=h(1,1)=q-1, \quad g(1, x)=q^{x}-1, \\
& h(1, x)=q^{x}-(q-1) x-1, \quad x>1 .
\end{aligned}
$$

(последнее получается из формулы $h(1, x)=g(1, x)-p(1, x)$, если заметить, что $p(1, x)=(q-1) x$ при $x>1)$.

По формуле (1) имеем

$$
c_{t}^{(n)}(m, n)=\left(\begin{array}{c}
m \\
t-n
\end{array}\right) h(m+n-t, n) .
$$

Далее, из (1) следует, что

$$
c_{t}^{(s)}(m, n) \leqslant\left(\begin{array}{c}
m \\
r
\end{array}\right)\left(\begin{array}{c}
n \\
s
\end{array}\right) g(n-s, r) q^{(m-r) n},
$$

$r=t-s$. Отсюда при $s=n-1$, учитывая (22), получаем:

$$
c_{t}^{(n-1)}(m, n) \leqslant\left(\begin{array}{c}
m \\
t-n+1
\end{array}\right) n\left(q^{t-n+1}-1\right) q^{m n-n(t-n)-n} .
$$

Точно таким же способом, используя (1) и (22), приходим к соотношениям

$$
\begin{gathered}
c_{t}^{(t-n+1)}(m, n) \\
\leqslant\left\{\begin{array}{c}
\left(\begin{array}{c}
n \\
t-n+1
\end{array}\right) q^{t-n+1} q^{m n-n(t-n)-n}, \quad m=n-1, \\
\left(\begin{array}{c}
n \\
t-n+1
\end{array}\right) n\left[q^{t-n+1}-(q-1)(t-n+1)-1\right] \\
\times q^{n^{2}-n(t-n)-n}, \quad m=n, \\
\left(c_{t}^{(t-n+1)}(m, n)=0 \quad \text { при } m<n-1\right)
\end{array}\right.
\end{gathered}
$$


и

$$
\begin{gathered}
c_{t}^{(t-n)}(m, n)=\left(\begin{array}{c}
n \\
t-n
\end{array}\right) g(2 n-t, n) \\
\left(c_{t}^{(t-n)}(m, n)=0 \quad \text { при } m<n\right) .
\end{gathered}
$$

Слагаемые в суммах $\Sigma_{1}, \Sigma_{2}, \Sigma_{3}$ оцениваем с помощью (4). Тогда

$$
\Sigma_{1} \leqslant \sum_{s=t-\sqrt{t}}^{n-2}\left(\begin{array}{l}
t \\
s
\end{array}\right)^{2} q^{m n-s(t-s)} .
$$

Отсюда по следствию леммы 3 , полагая в (15) $v=2$, находим, что

$$
\Sigma_{1}=o\left(q^{m n-n(t-n)-n}\right)
$$

Далее, также по (4)

$$
\Sigma_{2} \leqslant \sum_{s=\sqrt{t}}^{t-\sqrt{t}-1}\left(\begin{array}{l}
t \\
s
\end{array}\right)^{2} q^{m n-s(t-s)} .
$$

Отсюда по лемме 4 , полагая в $(17) l(n, t)=-n$, получаем, что

$$
\Sigma_{2}=o\left(q^{m n-n(t-n)-n}\right) \text {. }
$$

Наконец,

$$
\Sigma_{3} \leqslant \sum_{s=a}^{\sqrt{t}-1}\left(\begin{array}{l}
t \\
s
\end{array}\right)^{2} q^{m n-s(t-s)},
$$

где $a=\max \{t-m, t-n+2\}$. K правой части этого неравенства при $m=n-1, n$ применяем лемму 3 , а при $m \leqslant n-2$ следствие 2 леммы 3 , полагая в первом случае в (12) $p_{1}=p=n-2$, а во втором - беря в (16) $v=2$. В результате получаем, что

$$
\Sigma_{3}=o\left(q^{m n-n(t-n)-n}\right) .
$$

Складьвая неравенства (23)-(26) и равенства (27)-(29), приходим к соотношениям

$$
\begin{aligned}
c_{t}(m, n) \leqslant & \left(\begin{array}{c}
m \\
t-n
\end{array}\right) h(m+n-t, n) \\
& +\beta(m, n, t) q^{m n-n(t-n)-n}(1+o(1)), \quad m<n \\
c_{t}(n, n) \leqslant & \left(\begin{array}{c}
n \\
t-n
\end{array}\right)(h(2 n-t, n)+g(2 n-t, n)) \\
& +\beta(n, n, t) q^{n^{2}-n(t-n)-n}(1+o(1)) .
\end{aligned}
$$

Учитьвая, что $g(m+n-t, n), h(m+n-t, n) \leqslant q^{m n-n(t-n)}$, отсюда получаем неравенство (18). 
ЛЕмма 6. Справедливы следующие асимптотические при $n \rightarrow \infty$ соотношения:

а) если $m<n-1$, mo

$$
p(m, n) \leqslant(q-1) m n q^{m n-n}(1+o(1))
$$

б) если $m=n$, mo

$$
p(n, n) \leqslant\left((q-1) n^{2}+n\right) q^{n^{2}-2 n}(1+o(1)) .
$$

ДоказАтельство. Напомним, что $p(m, n)=c_{n}(m, n), p^{(s)}(m, n)=$ $c_{n}^{(s)}(m, n)$, и потому

$$
p(m, n)=\sum_{s=\max \{n-m, 1\}}^{n-1} p^{(s)}(m, n)=\sum_{s=1}^{n-1} c_{n}^{(s)}(m, n) .
$$

Аналогично доказательству леммы 5, запишем

$$
p(m, n)=c_{n}^{(n-1)}(m, n)+\Sigma_{1}+\Sigma_{2}+\Sigma_{3}+c_{n}^{(1)}(m, n),
$$

где

$$
\begin{gathered}
\Sigma_{1}=\sum_{s=n-\sqrt{n}}^{n-2} c_{n}^{(s)}(m, n), \quad \Sigma_{2}=\sum_{s=\sqrt{n}}^{n-\sqrt{n}-1} c_{n}^{(s)}(m, n), \\
\Sigma_{3}=\sum_{s=2}^{\sqrt{n}-1} c_{n}^{(s)}(m, n)
\end{gathered}
$$

$\left(c_{n}^{(1)}(m, n) \neq 0\right.$ лишш при $\left.m=n-1\right)$. Применяем формулу (1) и получаем, что

$$
c_{n}^{(n-1)}(m, n) \leqslant(q-1) m n q^{m n-n},
$$

а также при $m=n-1$

$$
c_{n}^{(1)}(m, n) \leqslant q n q^{n^{2}-2 n} .
$$

Далее, применяя к $\Sigma_{1}, \Sigma_{3}$ лемму 3 и ее следствия при $v=2$, а к $\Sigma_{2}$ лемму 4 при $l(n, t)=-n$, находим, что каждая из сумм $\Sigma_{1}, \Sigma_{2}, \Sigma_{3}$ есть $o\left(q^{m n-n}\right)$. Отсюда и из (34), (35) следует утверждение леммы.

Из полученных результатов без труда получаются асимптотические соотношения для чисел $f(m, n)$ и $h(m, n)$.

Следующее утверждение было установлено в [2] с применением принципа включения-исключения. 
ЛЕмма 7. При $n \rightarrow \infty$ справедливы следующие асимптотические соотношения:

$$
f(m, n) \leqslant \begin{cases}m q^{m n-n}(1+o(1)), & \text { если } m<n, \\ 2 n q^{n^{2}-n}(1+o(1)), & \text { если } m=n .\end{cases}
$$

ДокАЗАТЕльство. Пользуемся представлением

$$
f(m, n)=c_{n+1}(m, n)+\cdots+c_{n+4}(m, n)+\sum_{t_{1}=n+5}^{m+n} c_{t_{1}}(m, n),
$$

числа $m, n, t=n+k$ при любом фиксированном целом $k \geqslant 0$ удовлетворяют условию $(*)$. По лемме 5

$$
c_{n+k}(m, n) \leqslant\left\{\begin{array}{l}
\left(\begin{array}{c}
m \\
k
\end{array}\right) q^{m n-k n}(1+o(1)) \text { при } m<n, \\
2\left(\begin{array}{l}
n \\
k
\end{array}\right) q^{n^{2}-k n}(1+o(1)) \quad \text { при } m=n .
\end{array}\right.
$$

Таким образом, $c_{n+k}(m, n)=o\left(q^{m n-n}\right)$ при $n \rightarrow \infty$ для $k=2,3,4$.

Сумму остальных членов в (36) оцениваем по лемме 2 , полагая в (8) $v=1, u=5, t=n+1$ :

$$
\sum_{t_{1}=n+5}^{m+n} c_{t_{1}}(m, n)=O\left(m q^{m n-2 n}\right)=o\left(q^{m n-n)} .\right.
$$

Приходим, таким образом, к утверждению леммы.

Из леммы 7 следует известное (см. [3] при $q=2$ ) соотношение для числа матриц с ненулевым перманентом:

$$
g(m, n)=q^{m n}(1+o(1)), \quad m \leqslant n,
$$

при $n \rightarrow \infty$.

ТЕорема 1. а) Для числа $p(m, n)$ частично разложимых неотрицательных матрии размера $m \times n$ с ненулевым перманентом справедливы при $n \rightarrow \infty$ следующие асимптотические формулы:

$$
\begin{array}{ll}
p(m, n)=(q-1) m n q^{m n-n}(1+o(1)), & m<n-1, \\
p(m, n)=n((q-1) n+1) q^{m n-n}(1+o(1)), & m=n-1 .
\end{array}
$$

б) Для числа $h(m, n)$ полу-вполне неразложимых неотричательных матрии размера $m \times n$ справедливы при $n \rightarrow \infty$ следующ, асимптотические формуль:

$$
\begin{aligned}
& h(m, n)=q^{m n}-m((q-1) n+1) q^{m n-n}(1+o(1)), \quad m<n-1, \\
& h(m, n)=q^{n^{2}-n}-\left((q-1) n^{2}+2 n-1\right) q^{n^{2}-2 n}(1+o(1)), \quad m=n-1 .
\end{aligned}
$$


ДоКАЗАТЕЛЬСТво. Каждая полу-вполненеразложимая матрица имеет нульность не больше $n-1$. Поэтому ввиду (3),

$$
h(m, n)=g(m, n)-p(m, n) .
$$

Следовательно, по лемме 6 и соотношению (37)

$$
h(m, n)=q^{m n}(1+o(1)) .
$$

Далее, имеем $c_{n}(m, n) \geqslant c_{n}^{(n-1)}(m, n)$. Пусть $m<n-1$. Тогда (см. (1))

$$
c_{n}^{(n-1)}(m, n)=(q-1) m n q^{m-1} h(m-1, n-1),
$$

так как $g(1,1)=q-1$. Следовательно, ввиду $(42), c_{n}(m, n) \geqslant(q-1) m n \times$ $q^{m n-n}(1+o(1))$. Сравнение этого неравенства с противоположным по знаку неравенством (32) в лемме 6 дает формулу (38).

Пусть теперь $m=n-1$. Тогда

$$
c_{n}^{(1)}(n-1, n)=n h(0,1) g(n-1, n-1)=n g(n-1, n-1) .
$$

Следовательно,

$$
\begin{aligned}
c_{n}(n-1, n) & \geqslant c_{n}^{(n-1)}(n-1, n)+c_{n}^{(1)}(n-1, n) \\
& =(q-1)(n-1) n q^{n-2} h(n-2, n-1)+n g(n-1, n-1) .
\end{aligned}
$$

Отсюда, учитывая (37) и (42), а также соотношение (33) в лемме 6 , приходим к формуле (39).

Перейдем к оценке $h(m, n)$. Ясно, что $f(m, n) \geqslant c_{n+1}^{(n)}(m, n)=$ $m h(m-1, n)$. Учитывая $(42)$, получаем $f(m, n) \geqslant m q^{m n-n}(1+o(1))$, что вместе с леммой 7 приводит к

$$
f(m, n) \sim m q^{m n-n},
$$

если $n \rightarrow \infty$. Поэтому при $m \leqslant n-1$

$$
\begin{aligned}
& g(m, n)=q^{m n}-m q^{m n-n}(1+o(1)), \\
& h(m, n)=g(m, n)-p(m, n)=q^{m n}-p(m, n)-m q^{m n-n}(1+o(1)) .
\end{aligned}
$$

Из полученной вьше асимптотики для $p(m, n)$ теперь следуют формулы (40) и (41). Теорема доказана.

В следуюшей теореме мы установим асимптотическое поведение чисел $c_{t}(m, n)$ при $t>n$. Введем обозначение: если $m \leqslant n<t$, то пусть

$$
\gamma(m, n, t)=\left\{\begin{array}{l}
\left(\begin{array}{c}
m \\
t-n
\end{array}\right)(m+n-t)((q-1) n+1), \quad m<n, \\
\left(\begin{array}{c}
n \\
t-n
\end{array}\right)(2 n-t)((q-1) n+2), \quad m=n, \quad t>n+1, \\
(q-1) n^{3}+3 n^{2}-2 n, \quad m=n, \quad t=n+1 .
\end{array}\right.
$$


Теорема 2. Пусть $m, n, t$ удовлетворяют условию $(*) u t>n$. Для числа $c_{t}(m, n)$ неотрицательных матрии размера $m \times n$ и нульности $t$ справедливо при $n \rightarrow \infty$ следующее асимптотическое равенство:

$$
c_{t}(m, n)=\alpha q^{m n-n(t-n)}+(\beta-\gamma) q^{m n-n(t-n)-n}(1+o(1)),
$$

əде

$$
\alpha=\alpha(m, n, t)= \begin{cases}\left(\begin{array}{c}
m \\
t-n
\end{array}\right), & m<n, \\
2\left(\begin{array}{c}
n \\
t-n
\end{array}\right), & m=n,\end{cases}
$$

a $\beta=\beta(m, n, t) u \gamma=\gamma(m, n, t)$ находятся по формулам (20) $и$ (44) соответственно.

ДоказАТЕЛьСтво. Справедливо неравенство

$$
c_{t}(m, n) \geqslant c_{t}^{(n)}(m, n)+c_{t}^{(n-1)}(m, n)+c_{t}^{(t-n+1)}(m, n)+c_{t}^{(t-n)}(m, n) ;
$$

при этом, если $m<n$, то последнее слагаемое, а если $m<n-1$, то два последних слагаемых равны нулю. Согласно (1) при $r=t-n, s=n$ имеем

$$
c_{t}^{(n)}(m, n)=\left(\begin{array}{c}
m \\
t-n
\end{array}\right) h(m+n-t, n) .
$$

Далее, полагая в (1) $r=t-n+1, s=n-1$ и в $(22) x=t-n+1$, получаем $c_{t}^{(n-1)}(m, n)=\left(\begin{array}{c}m \\ t-n+1\end{array}\right) n q^{m+n-t-1}\left(q^{t-n+1}-1\right) h(m+n-t-1, n-1)$.

Так как $m+n-t-1 \leqslant n-1$, отсюда по теореме 1 находим, что если $n \rightarrow \infty$, то

$$
c_{t}^{(n-1)}(m, n)=\left[\left(\begin{array}{c}
m \\
t-n+1
\end{array}\right) n\left(q^{t-n+1}-1\right)\right] q^{m n-n(t-n)-n}(1+o(1)) .
$$

Рассмотрим отдельно два случая.

a) $m<n$. Если $m=n-1$, то, полагая в (1) $s=t-n+1, r=n-1$, получаем, что

$$
c_{t}^{(t-n+1)}(n-1, n)=\left(\begin{array}{c}
n \\
t-n+1
\end{array}\right) g(2 n-t-1, n-1),
$$

откуда, ввиду (37), следует, что

$$
c_{t}^{(t-n+1)}(m, n)=\left[\left(\begin{array}{c}
n \\
t-n+1
\end{array}\right) q^{t-n+1}\right] q^{n^{2}-n(t-n)-n}(1+o(1)) .
$$


Складьвая правые части в (47) и (48), а при $m=n-1$ в (47)-(49), получаем из (46), что

$$
c_{t}(m, n) \geqslant\left(\begin{array}{c}
m \\
t-n
\end{array}\right) h(m+n-t, n)+\beta q^{m n-n(t-n)-n}(1+o(1)) .
$$

Однако, выше мы доказали неравенство, противоположное по знаку (см. (30)). Следовательно,

$$
c_{t}(m, n)=\left(\begin{array}{c}
m \\
t-n
\end{array}\right) h(m+n-t, n)+\beta q^{m n-n(t-n)-n}(1+o(1))
$$

при $n \rightarrow \infty$. Применяем теперь теорему 1 , учитьвая, что $m+n-t<n-1$ при $m<n<t$. Поэтому $h(m+n-t, n)$ выражается по формуле (40), и мы приходим к утверждению теоремы.

б) $m=n$. Полагая в (1) $m=n, s=t-n, r=n$, получаем

$$
c_{t}^{(t-n)}(n, n)=\left(\begin{array}{c}
n \\
t-n
\end{array}\right) g(2 n-t, n) .
$$

Полагая в (1) $m=n, s=t-n+1, r=n-1$ и в (22) $x=t-n+1$, находим также, что

$$
\begin{aligned}
c_{t}^{(t-n+1)}(n, n)= & n\left(\begin{array}{c}
n \\
t-n+1
\end{array}\right) q^{2 n-t-1} \\
& \times\left(q^{t-n+1}-(q-1)(t-n+1)-1\right) g(2 n-t-1, n-1),
\end{aligned}
$$

откуда ввиду (37) следует, что

$$
\begin{aligned}
c_{t}^{(t-n+1)}(n, n)= & n\left(\begin{array}{c}
n \\
t-n+1
\end{array}\right)\left(q^{t-n+1}-(q-1)(t-n+1)-1\right) \\
& \times q^{n^{2}-n(t-n)-n}(1+o(1)) .
\end{aligned}
$$

Складьвая (47), (48), (50) и (51), получаем из (46), что

$$
\begin{aligned}
c_{t}(n, n) \geqslant & \left(\begin{array}{c}
n \\
t-n
\end{array}\right)(h(2 n-t, n)+g(2 n-t, n)) \\
& +\beta q^{n^{2}-n(t-n)-n}(1+o(1)) .
\end{aligned}
$$

Однако, выше мы доказали неравенство, противоположное по знаку (см. (31)). Следовательно,

$$
\begin{aligned}
c_{t}(n, n)= & \left(\begin{array}{c}
n \\
t-n
\end{array}\right)(h(2 n-t, n)+g(2 n-t, n)) \\
& +\beta q^{n^{2}-n(t-n)-n}(1+o(1)) .
\end{aligned}
$$


Так как $2 n-t<n$, из доказанного вьше соотношения (43) вытекает, что

$$
g(2 n-t, n)=q^{n^{2}-n(t-n)}-(2 n-t) q^{n^{2}-n(t-n)-n}(1+o(1)) .
$$

Если $t>n+1$, то $2 n-t<n-1$ и $h(2 n-t, n)$ выражается по формуле (40):

$$
\begin{aligned}
h(2 n-t, n)= & q^{n^{2}-n(t-n)} \\
& -(2 n-t)((q-1) n+1) q^{n^{2}-n(t-n)-n}(1+o(1)) .
\end{aligned}
$$

При $t=n+1 h(2 n-t, n)$ выражаем по формуле (41):

$$
h(n-1, n)=q^{n^{2}-n}-\left((q-1) n^{2}+2 n-1\right) q^{n^{2}-2 n}(1+o(1)) .
$$

Учитывая (53)-(55), в каждом из случаев приходим к утверждению теоремы.

СЛЕДСТВИЕ. Для числа $f(n, n)$ матрии, порядка $n$ с нулевым перманентом справедливо при $n \rightarrow \infty$ следующее асимптотическое разлохсение:

$$
\begin{array}{rl}
f(n, n)=2 & n q^{n^{2}-n} \\
& +\left[(q-1)^{2} n^{3}-\left(q^{2}-q+2\right) n^{2}+n\right] q^{n^{2}-2 n}(1+o(1)) .
\end{array}
$$

Действительно,

$$
f(n, n)=c_{n+1}(n, n)+c_{n+2}(n, n)+\cdots+c_{n+5}(n, n)+\sum_{t_{1}=n+6}^{2 n} c_{t_{1}}(n, n)
$$

Согласно доказанной теореме и лемме 2 (беря в $(8) t=n+1, v=2$ )

$$
\begin{aligned}
f(n, n)= & c_{n+1}(n, n)+c_{n+2}(n, n)+o\left(q^{n^{2}-2 n}\right), \\
c_{n+1}(n, n)= & 2 n q^{n^{2}-n} \\
& +\left[(q-1)^{2} n^{3}-\left(q^{2}-q+3\right) n^{2}+2 n\right] q^{n^{2}-2 n}(1+o(1)), \\
c_{n+2}(n, n)= & \left(n^{2}-n\right) q^{n^{2}-2 n}(1+o(1)) .
\end{aligned}
$$

Таким образом, приходим к требуемому результату.

Следствие демонстрирует другой способ получения асимптотического разложения для $f(n, n)$, чем в [2] (формула (56) дает два старших члена разложения для $f(n, n))$. 


\section{§2. Вполне неразложимые матрицы}

В теореме $1 \S 1$ указываются старшие члены асимптотического разложения при $n \rightarrow \infty$ для числа $h(m, n)$ полу-вполне неразложимых неотрицательных матрищ размера $m \times n$ при $m \leqslant n-1$. На случай $m=n$, т.е. при рассмотрении вполне неразложимых матриц, рассуждения в 1 непосредственно не переносятся, так как при $t=n$ тогда не имеет места формула (1). В этом параграфе мы покажем, как можно обойти это затруднение, и получим асимптотическое разложение для числа $h(n, n)$ вполне неразложимых матриц при $n \rightarrow \infty$.

Лемма 8. Пусть $s$ - натуральное число, $1 \leqslant s \leqslant n-1$. Любая матрича $A \in M_{n}^{(s)}(n, n)$ nри $s>n / 2$ содержит единственную максимальную подматрииу. При $s \leqslant n / 2$ A содержит либо одну, либо две максимальные подматрицы; в последнем случае общее число строк, в которых расположены әлементы как одной, так и другой максимальной подматрицы, равно $n-2 s$.

ДокАЗАТЕльство. Как показано в [2] (следствие леммы 1), любые две максимальные подматрицы в неотрицательной матрице либо совпадают, либо не пересекаются. Если $s>n / 2$, то предположение о том, что в $A$ имеются две максимальные подматрицы, ведет к противоречию с тем, что $A$ принадлежит $M_{n}^{(s)}(n, n)$.

Пусть теперь $s \leqslant n / 2$ и матриша $A$ имеет две максимальные подматрицы $M$ и $M_{1}$. Обозначим $r=n-s$. Если число строк, в которых расположены нули как $M$, так и $M_{1}$, равно $x$, то $A$ содержит нулевую подматрицу размера $x \times 2 s$. Так как $2 r-x \leqslant n$, то $x \geqslant 2 r-n=n-2 s$. Если $x>n-2 s$, то $x+2 s>n$, что противоречит тому, что нульность $A$ равна $n$. Итак, $x=n-2 s$. Ясно поэтому, что еще одной максимальной подматрицы в $A$, отличной от $M$ и $M_{1}$, быть не может. Лемма доказана.

Лемма 9. Пусть $A$ - матрица из $M_{n}^{(s)}(n, n), 1 \leqslant s \leqslant n-1$. Тогда $A$ перестановочно эквивалентна матриче следующего вида:

$$
\left(\begin{array}{cc}
A_{1} & O_{r s} \\
A_{3} & A_{2}
\end{array}\right)
$$

əде $r=n-s, O_{r s}-$ нулевая матрица размера $r \times s, A_{1}-$ матрица порядка $r$ с ненулевым перманентом, $A_{2}$ - вполне неразложимая матрича порядка $s$.

ДоКАЗАТЕЛЬСТВО получается дословным переносом на случай $m=n$ доказательства теоремы 4 работы [2]. 
Пусть $s \leqslant n / 2$ - натуральное число. Множество матриц $P_{n}^{(s)}=$ $M_{n}^{(s)}(n, n)$ разобьем на два подмножества $P_{n 1}^{(s)}$ и $P_{n 2}^{(s)}:$ к первому отнесем все матрицы с единственной максимальной подматрицей, ко второму - все матрицы с двумя максимальньми подматрицами. Положим $c_{n 1}^{(s)}=\left|P_{n 1}^{(s)}\right|$, $c_{n 2}^{(s)}=\left|P_{n 2}^{(s)}\right|$. Тогда $c_{n}^{(s)}(n, n)=c_{n 1}^{(s)}+c_{n 2}^{(s)}$. Далее, пусть

$$
\bar{c}_{n}^{(s)}=q^{s(n-s)}\left(\begin{array}{l}
n \\
s
\end{array}\right)^{2} g(n-s, n-s) h(s, s) .
$$

Ясно, что $c_{n}^{(s)}(n, n) \leqslant \bar{c}_{n}^{(s)}$, так как любая матрица из $P_{n}^{(s)}$ перестановочно эквивалентна матрице вида (57) из леммы 9.

ЛЕмма 10. Пусть $n, s$ - натуральные числа, $s \leqslant n / 2, B, C-$ вполне неразложимые матрицы порядков $r=n-s$ и $s$ соответственно, $D$ - произвольная ненулевая матрица размера $s \times r$. Тогда матрица

$$
A=\left(\begin{array}{cc}
B & O_{r s} \\
D & C
\end{array}\right)
$$

принадлежат подмножеству $P_{n 1}^{(s)}$.

ДоКАЗАТЕЛЬСТво. Пусть $M$ - максимальная подматрица матрицы $A$ и ее размер $-r^{\prime} \times s^{\prime}, r^{\prime}+s^{\prime} \geqslant n$. Так как $D-$ ненулевая матрица, то $M$ не содержится в $D$. Обозначим $M_{1}$ и $M_{2}$ пересечения $M$ соответственно с $B$ и $C$ и пусть их размеры $-r_{1}^{\prime} \times s_{1}^{\prime}$ и $r_{2}^{\prime} \times s_{2}^{\prime}$. Тогда $r_{1}^{\prime}+s_{1}^{\prime} \leqslant r-1$, $r_{2}^{\prime}+s_{2}^{\prime} \leqslant s-1$ по условиям леммы. Отсюда следует, во-первых, что матрица $M_{2}$ пуста, так как в противном случае $M$ имела бы нульность не больше $r+s-1=n-1$ и не могла бы быть максимальной матрицей. Далее, $M$ не может быть расположена в первых $r$ столбцах, так как всякая такая матрица имеет нульность не больше $r-1+s=r-1+n-r=n-1$. Следовательно, $M$ содержится в первых $r$ строках. Но тогда $M$ должна совпадать с матрицей, расположенной в правом верхнем углу. Лемма доказана.

СЛЕДСТВИЕ.

$$
c_{n 1}^{(s)} \geqslant\left(\begin{array}{l}
n \\
s
\end{array}\right)^{2} h(s, s) h(n-s, n-s)\left(q^{s(n-s)}-1\right)
$$

$n p u s \leqslant n / 2$.

Действительно, подмножеству $P_{n 1}^{(s)}$ принадлежит всякая матрища, перестановочно эквивалентная матрице вида (58). 
Лемма 11. Пусть $n, s$ - натуральные числа, $s \leqslant n / 2$ и матрица $A$ принадлежит множеству $P_{n 2}^{(s)}$. Тогда $A$ перестановочно әквивалентна матрице вида

$$
A^{\prime}=\left(\begin{array}{cc}
B & O_{r s} \\
& C
\end{array}\right)
$$

где $r=n-s, C-$ вполне неразложимая матрица порядка $s$, а $B$ перестановками столбчов и первых $г$ строк может быть переведена в матрииу

$$
\left(\begin{array}{cc}
B_{3} & B_{2} \\
B_{1} & O_{r s}
\end{array}\right)
$$

c матрицей $B_{1}$ размера $r \times(r-s)$ и матрицей $B_{2}$ порядка s такими, что per $B_{1}^{T} \neq 0$, per $B_{2} \neq 0$.

ДоказАтельство. По лемме 9 всякая матрица $A$ из $P_{n}^{(s)}$ перестановочно эквивалентна матрице вида (60) со вполне неразложимой матрицей $C$ порядка $s$. Так как $A \in P_{n 2}^{(s)}$, в подматрице $B$ должна содержаться вторая максимальная подматрица $M^{\prime}$ матрицы $A$, при этом по лемме 8 в точности $n-2 s$ строк матрицы $M^{\prime}$ находятся среди первых $n-s$ строк $A^{\prime}$. Следовательно, дальнейшими перестановками $n-s$ первых столбцов и $n-s$ первых строк в матрице $A^{\prime}$ можно подматрицу $B$ привести к виду (61). Так как нульность $A$ равна $n$, то, очевидно, per $B_{2} \neq 0$, per $B_{1}^{T} \neq 0$. Лемма доказана.

СлЕДСТВИЕ. Справедливо неравенство

$$
c_{n 2}^{(s)} \leqslant q^{s(n-s)}\left(\begin{array}{l}
n \\
s
\end{array}\right)^{2}\left(\begin{array}{c}
n-s \\
s
\end{array}\right)^{2} h(s, s) g(s, s) g(n-2 s, n-s)
$$

npu $s \leqslant n / 2$.

Перейдем теперь к оценке величины $p(n, n)=c_{n}(n, n)$. Из леммы 9 и формулы (1) следуют неравенства

$$
\begin{aligned}
& c_{n}^{(n-1)}(n, n)=q^{n-1} n^{2} g(1,1) h(n-1, n-1) \leqslant(q-1) n^{2} q^{n^{2}-n}, \\
& c_{n}^{(n-2)}(n, n)=q^{2(n-2)}\left(\begin{array}{c}
n \\
2
\end{array}\right)^{2} g(2,2) h(n-2, n-2) \leqslant g(2,2)\left(\begin{array}{c}
n \\
2
\end{array}\right)^{2} q^{n^{2}-2 n},
\end{aligned}
$$

здесь, как легко заметить, $g(2,2)=q^{4}-4(q-1)^{2}-4(q-1)-1$. Далее, аналогично,

$$
\begin{aligned}
& c_{n}^{(1)}(n, n) \leqslant \bar{c}_{n}^{(1)} \leqslant(q-1) n^{2} q^{n^{2}-n}, \\
& c_{n}^{(2)}(n, n) \leqslant \bar{c}_{n}^{(2)} \leqslant h(2,2)\left(\begin{array}{l}
n \\
2
\end{array}\right)^{2} q^{n^{2}-2 n},
\end{aligned}
$$


здесь, очевидно, $h(2,2)=(q-1)^{4}$.

Пусть $S=\sum_{s=3}^{n-3} c_{n}^{(s)}(n, n)$. Тогда

$$
c_{n}(n, n) \leqslant c_{n}^{(n-1)}(n, n)+c_{n}^{(n-2)}(n, n)+\bar{c}_{n}^{(1)}+\bar{c}_{n}^{(2)}+S .
$$

Оцениваем $S$ с помощью леммы 3 при $v=3$ и леммы 4 . Получаем тогда, что при $n \rightarrow \infty$

$$
\begin{aligned}
p(n, n)= & c_{n}(n, n) \leqslant 2(q-1) n^{2} q^{n^{2}-n} \\
& +\left(\begin{array}{l}
n \\
2
\end{array}\right)^{2}(g(2,2)+h(2,2)) q^{n^{2}-2 n}+o\left(q^{n^{2}-2 n}\right),
\end{aligned}
$$

т.e.

$$
p(n, n) \leqslant 2(q-1) n^{2} q^{n^{2}-n}(1+\varphi(n)),
$$

где $\varphi(n)=O\left(n^{2} q^{-n}\right)$.

Согласно (56)

$$
g(n, n)=q^{n^{2}}-f(n, n)=q^{n^{2}}\left(1+O\left(n q^{-n}\right)\right),
$$

поэтому из (63) следует, что

$$
h(n, n)=g(n, n)-p(n, n)=q^{n^{2}}\left(1+O\left(n^{2} q^{-n}\right)\right)
$$

при $n \rightarrow \infty$.

ТЕОРема 3. Пусть $s-$ произвольное натуральное число, $1 \leqslant s \leqslant n-1$. Тогда

$$
c_{n}^{(s)}(n, n)=c_{n 1}^{(s)}\left(1+O\left(n^{2} q^{-n}\right)\right),
$$

если $n \rightarrow \infty$.

ДокАЗАТЕЛЬСтво. Если $s>n / 2$, то согласно лемме $8 c_{n 2}^{(s)}=0$, $c_{n}^{(s)}(n, n)=c_{n 1}^{(s)}$, т.е. $(66)$ выполняется. Пусть $s \leqslant n / 2$. Имеем

$$
c_{n}^{(s)}(n, n)=c_{n 1}^{(s)}+c_{n 2}^{(s)}=c_{n 1}^{(s)}\left(1+\frac{c_{n 2}^{(s)}}{c_{n 1}^{(s)}}\right) .
$$

По следствиям из лемм 10 и 11 (см. (59) и (63))

$$
\frac{c_{n 2}^{(s)}}{c_{n 1}^{(s)}} \leqslant \frac{q^{s(n-2 s)}\left(\begin{array}{c}
n \\
s
\end{array}\right)^{2}\left(\begin{array}{c}
n-s \\
s
\end{array}\right)^{2} h(s, s) g(s, s) g(n-2 s, n-s)}{\left(q^{s(n-s)}-1\right)\left(\begin{array}{c}
n \\
s
\end{array}\right)^{2} h(s, s) h(n-s, n-s)} .
$$


Заметим, что $q^{s(n-2 s)} g(s, s) /\left(q^{s(n-s)}-1\right) \leqslant 1$, а также, что ввиду (64) и $(65)$

$$
\begin{aligned}
g(n-2 s, n-s) & =q^{(n-s)(n-2 s)}(1+o(1)), \\
h(n-s, n-s) & =q^{(n-s)^{2}}(1+o(1)) .
\end{aligned}
$$

Поэтому асимптотическое поведение правой части (67) определяется отношением

$$
\frac{\left(\begin{array}{c}
n-s \\
s
\end{array}\right)^{2}}{q^{s n-s^{2}}} \text {. }
$$

Покажем, что оно представляет собой величину $O\left(n^{2} q^{-n}\right)$. При $s=1,2$ это очевидно. Пусть $s \geqslant 3$. Тогда ввиду (5)

$$
\left(\begin{array}{c}
n-s \\
s
\end{array}\right)^{2}=O\left(\frac{2^{2(n-s)}}{n-s}\right)
$$

при $n \rightarrow \infty$, и

$$
\frac{\left(\begin{array}{c}
n-s \\
s
\end{array}\right)^{2}}{q^{s n-s^{2}}}=O\left(\frac{1}{(n-s) q^{(s-2)(n-s)}}\right)=o\left(q^{-n}\right) .
$$

Таким образом, $c_{n 2}^{(s)} / c_{n 1}^{(s)}=O\left(n^{2} q^{-n}\right)$, и теорема доказана.

TEOPEMA 4. Прu $n \rightarrow \infty$

$$
p(n, n)=2(q-1) n^{2} q^{n^{2}-n}\left(1+O\left(n^{2} q^{-n}\right)\right) .
$$

ДоКАЗАТЕЛЬСТВО. Воспользуемся очевидными неравенствами

$$
\begin{aligned}
p(n, n) & \geqslant c_{n}^{(n-1)}(n, n)+c_{n}^{(1)}(n, n) \\
& \geqslant c_{n}^{(n-1)}(n, n)+c_{n 1}^{(1)} .
\end{aligned}
$$

Далее, по лемме 8 и следствию леммы 10

$$
\begin{gathered}
c_{n}^{(n-1)}(n, n)=q^{n-1}(q-1) n^{2} h(n-1, n-1), \\
c_{n 1}^{(1)} \geqslant\left(q^{n-1}-1\right)(q-1) n^{2} h(n-1, n-1) .
\end{gathered}
$$

Так как $h(n-1, n-1)=q^{(n-1)^{2}}(1+\psi(n))$, где $\psi(n)=O\left(n^{2} q^{-n}\right)$ (см. (65)), отсюда следует, что $p(n, n) \geqslant 2(q-1) n^{2} q^{n^{2}-n}\left(1+\psi_{1}(n)\right)$, где $\psi_{1}(n)=O\left(n^{2} q^{-n}\right)$. Сопоставляя полученное неравенство с неравенством (63), получаем утверждение теоремы. 
Теорема 5. Для числа $h(n, n)$ вполне неразложсимых матрии порядка п справедливо следующее асимптотическое соотношение

$$
h(n, n)=q^{n^{2}}-2\left((q-1) n^{2}+n\right) q^{n^{2}-n}\left(1+O\left(n^{2} q^{-n}\right)\right)
$$

npu $n \rightarrow \infty$.

ДокаЗАТЕЛЬСТво. Имеем

$$
h(n, n)=q^{n^{2}}-f(n, n)-p(n, n) .
$$

Утверждение следует теперь из формулы (56) и теоремы 4.

Отметим в заключение, что результаты о строении частично разложимых и вполне неразложимых матриц (леммы 8-11) справедливы для матриц с произвольными неотрицательными элементами.

Математический институт

Поступило

им. В. А. Стеклова РАН

28.06 .95

\section{СПИСОК ЦИТИРОВАННОЙ ЛИТЕРАТУРЫ}

[1] Fennet T. I., Loizou G. Combinatorial aspects of rectangular non-negative matrices // Diserete Math. 1977. V. 20. P. 217-234.

[2] Болотников Ю. В., Тараканов В. Е. Неотрицательные матрицы с нулевым перманентом // Матем. заметки. 1995. Т. 58. № 4. С. 493-504.

[3] Сачков В. Н. Вероятностные методы в комбинаторном анализе. М.: Наука, 1978. 